\title{
Relation between parents' and children's smoking behaviour and attitudes
}

\author{
M MURRAY, S KIRYLUK, AND A V SWAN \\ From the Department of Community Medicine, St Thomas' Hospital Medical School, United Medical and \\ Dental Schools, London SE1 7EH
}

SUMMARY In the MRC/Derbyshire Smoking Study, a cohort of about 6000 adolescents was surveyed annually about their smoking behaviour, attitudes, and other issues from when they entered secondary school at 11-12 until 15-16 years and then again at 18-19 years. Their parents answered a similar questionnaire when their children were aged 11-12 and 15-16 years. In this paper we report the findings of an investigation focussed on the relation between parents' and childrens' smoking behaviour and attitudes at different stages of adolescence. It reveals substantial agreement between children's and parents' reports of parents' smoking behaviour and attitudes, that children from one-parent families are more likely than their peers to smoke, and that boys are more likely to smoke if their fathers smoke and girls if their mothers smoke. In addition, maternal attitudes were independently related to the boys' smoking behaviour. The implications of these findings for health education are discussed.

Twenty five years ago social researchers implicated parental smoking behaviour ${ }^{1}$ and parental permissiveness regarding smoking ${ }^{2}$ as being influential in the development of smoking among children and adolescents. Subsequently, several investigators have reported further details of these relations but there are a number of disagreements. Some reported a sex linking in the relation between parents' and children's smoking behaviour ${ }^{3}$ and others that this relation is stronger among girls than among boys. ${ }^{4}$ Some considered that parental attitudes are more important than their behaviour ${ }^{5}$ whereas others reported that attitudes were of little importance. $^{6}$

One possible reason for the inconsistency in these findings is that each of the studies had one or more methodological limitations. The studies were usually small and did not consider whether the relationship between parents and children changed during adolescence. In addition, although the findings were usually based on analyses of children's reports of their parents' smoking behaviour and attitudes, no attempt was made to measure the extent to which this differed from that of the parents' own reports. Finally, the studies were usually cross sectional in design and so could not reveal the effect of changing parental smoking behaviour and attitudes on their children.
In this paper we aim to identify more precisely the nature of parental influence on their children's smoking through an analysis of the data collected in a large longitudinal study of adolescent smoking behaviour.

\section{Method}

The data were taken from the MRC/Derbyshire Smoking Study. ${ }^{7}$ In that study a cohort of over 6000 adolescents was followed from entrance to secondary school aged 11-12 years in 1974 until they reached school leaving age in 1978. Each year the adolescents were required to answer, in their classrooms, a questionnaire about their smoking behaviour and other issues. In 1981, a questionnaire requesting details of their smoking behaviour was sent to all the adolescents from the cohort whose addresses were provided by their schools in 1978 . In 1974 and 1978 a questionnaire requesting details of their own smoking behaviour and attitudes towards smoking was sent to the parents of those adolescents who answered in those years.

The response rate of the adolescents fell from $86 \%$ of 7383 in 1974 to $75 \%$ of 7611 in 1978 and then rose to $79 \%$ of a restricted sample of 6983 in 1981 . The parents' response rate fell from $88 \%$ of 6330 in 1974 to $75 \%$ of 5686 in 1978 . The children's and their 
parents' replies were individually linked in 1974, then through to 1978 and on to 1981 . Of the 6310 11-12 year olds who answered the questions on smoking in 1974, 85\% had questionnaires answered by their parents in that year. Of these adolescents $68 \%$ were traced in 1978 and $55 \%$ in 1981.

In the questionnaire the parents scored 21 attitude items on a five-point scale of strongly agree to strongly disagree. For analysis the parents' replies in 1974 to the various questions about their attitudes towards smoking were grouped to form four composite variables to measure their attitudes towards the following:

(1) Smoking in general (ie, agreeing or disagreeing with "Smoking does more good for a person than harm", "There is nothing wrong with smoking", "Smoking is pleasurable", "Smoking is a dirty habit")

(2) Smoking by children (ie, (dis)agreeing with "Cigarettes should be less available to adolescents", "I wouldn't like my children to smoke", "Children should never smoke")

(3) Smokers' rights (ie, (dis)agreeing with "Morally, no one should prevent someone else from smoking", "Parents who smoke have no right to prevent their children from smoking")

(4) Smoking prevention (ie, (dis)agreeing with "Children should be discouraged from smoking", "There is not enough fuss being made about the dangers of smoking").

The results are in the form of a descriptive presentation of prevalences with regression analyses used to assess the significance of differences between various groups allowing for other factors. ${ }^{8}$ Unless otherwise stated significance tests are all at the $5 \%$ level.

\section{Results}

Table 1 gives a summary of the information on parents' smoking and attitudes from the 1974

Table 1 Parents' self-reported smoking behaviour and attitudes (1974)

\begin{tabular}{lcc}
\hline & $\begin{array}{l}\text { Fathers } \\
\%\end{array}$ & $\begin{array}{l}\text { Mothers } \\
\%\end{array}$ \\
\hline Smoker & $54 \cdot 5$ & $39 \cdot 4$ \\
Favourable attitude to smoking in general & 66.5 & 50.9 \\
Favourable attitude to youth smoking & $14 \cdot 3$ & $12 \cdot 8$ \\
Favourable attitude to smokers' rights & $65 \cdot 4$ & 65.5 \\
Unfavourable attitude to smoking prevention & $5 \cdot 8$ & $5 \cdot 2$ \\
Nmin & 2272 & 2380 \\
\hline
\end{tabular}

parents' questionnaire when their children were aged 11-12 years. Over half the fathers and about $40 \%$ of the mothers admitted smoking and about two thirdso of the parents held a favourable attitude to smoking in general and to smokers' rights, although less than $15 \%$ favoured children smoking and only $5 \%$ were against more health education campaigns.

Table 2 compares the data on parents' smoking $\frac{\text { T }}{\mathbb{D}}$ behaviour obtained directly with the data from the children's reports of their parents' smoking. behaviour. Taking the parents' reports as the truth, this shows that of the mothers who smoked, $96 \%$ o were correctly identified by their children as being을 smokers, while of the fathers who smoked, $92 \%$ were $\bar{s}$ correctly identified. However, of the mothers and $\propto$ fathers who claimed not to smoke, $10 \%$ were identified by their children as being smokers.

Table 2 Comparison of parents' reports of their smoking behaviour with children's reports of their parents' smoking behaviour

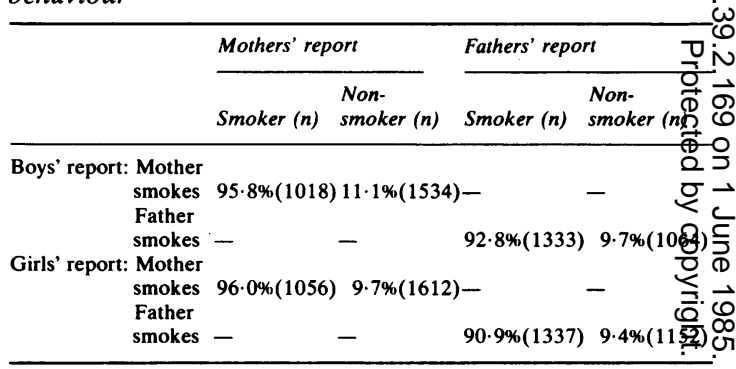

Although there was no directly equivalent question on parents' attitude to smoking on both parents' and children's questionnaires, it was possible to compare the mothers' reports of their attitudeô towards their children smoking with their own children's reports of parental permissiveness towards smoking. Table 3 shows that of those mothers who. reported that they would not like their children tod smoke, $78 \%$ of their sons and $69 \%$ of their daughters? reported that their parents did not allow them to smoke. This meant that roughly a quarter of the mothers who did not want their children to smokeo were not considered anti-smoking by their children. Conversely, of those mothers who were not againsto their children smoking, only a third of their children reported that their parents did not forbid them toㅡㅡㄹ smoke.

In $1974,91 \%$ of the children lived with both parents, $7 \%$ with mother alone, $2 \%$ with father alone and the remaining $1 \%$ in some institution. Whereas $51 \%$ of single mothers and $66 \%$ of single fatherso 
Table 3 Comparison of mothers' attitudes to their children smoking with children's reports of their parents' restrictions on smoking

\begin{tabular}{|c|c|c|c|}
\hline \multicolumn{2}{|c|}{$\begin{array}{l}\text { Child's report: } \\
\text { "My parents don't allow me to smoke" }\end{array}$} & \multicolumn{2}{|c|}{$\begin{array}{l}\text { Mother's report: } \\
\text { "I wouldn't like my child to smoke" }\end{array}$} \\
\hline & & Agree & Disagree/Don't know \\
\hline $\begin{array}{l}\text { Boys } \\
\text { Girls }\end{array}$ & $\begin{array}{l}\text { Agree } \\
\text { Disagree/Don't know } \\
\text { Agree } \\
\text { Disagree/Don't know }\end{array}$ & $\begin{array}{r}1890(78 \cdot 2 \%) \\
526(21 \cdot 8 \%) \\
1940(69 \cdot 2 \%) \\
863(30 \cdot 8 \%)\end{array}$ & $\begin{array}{r}139(66 \cdot 8 \%) \\
69(33 \cdot 2 \%) \\
126(67 \cdot 4 \%) \\
61(32 \cdot 6 \%)\end{array}$ \\
\hline
\end{tabular}

smoked in 1974 , only $38 \%$ of the other mothers and $53 \%$ of the other fathers smoked. Table 4 details the proportion of adolescents smoking in 1974, 1978, and 1981, when aged $11-12,15-16$, and $18-19$ years respectively, with reference to whether or not they lived with both parents in 1974. At each age those adolescents who came from single-parent families were most likely to smoke. The boys were most likely to smoke if they lived only with their mothers, and conversely the girls if they lived only with their fathers. This appeared as a significant interaction in the regression analysis. The relations were apparent irrespective of the parents' smoking behaviour.

Table 4 Smoking behaviour of adolescents in 1974, 1978, and 1981 who lived with one or both parents in 1974

\begin{tabular}{|c|c|c|c|c|c|c|}
\hline \multirow{2}{*}{$\begin{array}{l}\text { Parents present } \\
\text { in } 1974\end{array}$} & \multicolumn{2}{|l|}{1974} & \multicolumn{2}{|l|}{1978} & \multicolumn{2}{|l|}{1981} \\
\hline & $\%$ & No & $\%$ & No & $\%$ & No \\
\hline & \multicolumn{6}{|l|}{ Boys } \\
\hline Both parents & $5 \cdot 5$ & 2418 & $23 \cdot 0$ & 1802 & $31 \cdot 2$ & 1828 \\
\hline Mother only & $9 \cdot 2$ & 185 & $36 \cdot 4$ & 132 & $42 \cdot 4$ & 125 \\
\hline Father only & $8 \cdot 7$ & 46 & $16 \cdot 7$ & 30 & $40 \cdot 7$ & 27 \\
\hline \multirow[t]{2}{*}{ Other } & $5 \cdot 7$ & 35 & $11 \cdot 8$ & 17 & $40 \cdot 0$ & 15 \\
\hline & \multicolumn{6}{|l|}{ Girls } \\
\hline Both parents & $2 \cdot 2$ & 2528 & $21 \cdot 2$ & 1950 & $28 \cdot 8$ & 1974 \\
\hline Mother only & $2 \cdot 5$ & 200 & 26.7 & 135 & 29.9 & 144 \\
\hline Father only & $6 \cdot 5$ & 46 & $50 \cdot 0$ & 24 & $58 \cdot 3$ & 24 \\
\hline Other & $2 \cdot 9$ & 35 & $34 \cdot 8$ & 23 & $35 \cdot 0$ & 20 \\
\hline
\end{tabular}

Table 5 details the proportion of adolescents smoking in 1974, 1978, and 1981 with reference to their parental smoking behaviour and attitudes in 1974. Since information on both parents in 1974 was used in the statistical analysis those adolescents from one-parent families are not included in this table. A series of regression models ${ }^{8}$ was fitted to estimate the form and strength of the relation between parental smoking behaviour and attitudes in 1974 and the prevalence of smoking among the boys and girls in 1974,1978 , and 1981. The analyses not only considered the parental behaviour and attitudes simultaneously but they also took account of those variables already known to be associated with smoking which were social class, sibling smoking behaviour, and the children's friendship patterns. ${ }^{79}$ In this analysis several of the parental characteristics were found to be significantly related to the extent of adolescents smoking.

At each of the three ages the boys were significantly more likely to smoke if, when the boys were aged 11-12, their mothers were not opposed to children smoking (attitude 2), irrespective of the mothers' own smoking behaviour. The 11-12 year old boys were also significantly more likely to smoke if their mothers were not opposed to smoking in general (attitude 1). At 15-16 years the boys were significantly more likely to smoke if their mothers had smoked four years previously. Finally, at 15-16 and 18-19 years the boys were significantly more likely to smoke if their fathers had smoked when the boys were 11-12 years.

Among 11-12 and 18-19 year old girls smoking was significantly more likely if their mothers had smoked when the girls were 11-12 years. This. relation approached significance among 15-16 year old girls who were also significantly more likely to smoke if their fathers had smoked four years previously. There was no significant relation between parental attitudes and the girls' smoking behaviour.

Table 6 details the proportion of $15-16$ and 18-19 year olds smoking according to the change in their parental characteristics between 1974 and 1978 when the adolescents were aged between 11 and 16 years. The numbers available are considerably reduced because many mothers did not reply in 1978 . Since in 1978 the fathers were not required to answer the questionnaire, data on their attitudes were not available and information on their smoking behaviour was obtained from the mothers.

There was a consistent pattern in the marginal totals (table 6). Those adolescents whose parents moved in the direction of increasing the risk (eg, adopted smoking) were more likely to adopt smoking themselves than those whose parents did not (eg, remained non-smokers). This pattern was often delayed, being more apparent at 18-19 than at 15-16 years. However, the number of children whose parents' behaviour changed was small, and these patterns did not reach significance in the regression analysis.

\section{Discussion}

This study revealed substantial agreement between children and parents in reporting parental smoking behaviour. The slightly higher prevalence of parental smoking reported by the children may have been due 
Table 5 Proportion of adolescents smoking in 1974, 1978, and 1981 in relation to characteristics of their parents in 1974

\begin{tabular}{|c|c|c|c|c|c|c|c|c|c|c|c|c|}
\hline \multirow[b]{3}{*}{1974 parental characteristics } & \multicolumn{6}{|l|}{ Boys } & \multicolumn{6}{|l|}{ Girls } \\
\hline & \multicolumn{2}{|c|}{$1974(11-12 y r)$} & \multicolumn{2}{|c|}{$1978(15-16 y r)$} & \multicolumn{2}{|c|}{$1981(18-19 y r)$} & \multicolumn{2}{|c|}{$1974(11-12 y r)$} & \multicolumn{2}{|c|}{$1978(15-16 y r)$} & \multicolumn{2}{|c|}{1981 (18-19 yr) } \\
\hline & $\%$ & $\mathrm{No}$ & $\%$ & No & $\%$ & No & $\%$ & No & $\%$ & No & $\%$ & No \\
\hline $\begin{array}{l}\text { Father does not smoke } \\
\text { Father used to smoke } \\
\text { Father smokes }\end{array}$ & $\begin{array}{l}2 \cdot 5 \\
3 \cdot 9 \\
7 \cdot 1\end{array}$ & $\begin{array}{r}528 \\
540 \\
1280\end{array}$ & $\begin{array}{l}15 \cdot 5 \\
19 \cdot 4 \\
27 \cdot 6\end{array}$ & $\begin{array}{l}413 \\
422 \\
911\end{array}$ & $\begin{array}{l}22 \cdot 7 \\
29 \cdot 4 \\
35 \cdot 9\end{array}$ & $\begin{array}{l}409 \\
442 \\
931\end{array}$ & $\begin{array}{l}1.9 \\
1.9 \\
1.9\end{array}$ & $\begin{array}{r}585 \\
581 \\
1275\end{array}$ & $\begin{array}{l}14 \cdot 7 \\
19 \cdot 3 \\
25 \cdot 7\end{array}$ & $\begin{array}{l}496 \\
461 \\
930\end{array}$ & $\begin{array}{l}21 \cdot 7 \\
27 \cdot 5 \\
32 \cdot 6\end{array}$ & $\begin{array}{l}480 \\
458 \\
980\end{array}$ \\
\hline $\begin{array}{l}\text { Mother does not smoke } \\
\text { Mother used to smoke } \\
\text { Mother smokes }\end{array}$ & $\begin{array}{l}4 \cdot 1 \\
5 \cdot 6 \\
7 \cdot 6\end{array}$ & $\begin{array}{r}1203 \\
268 \\
909\end{array}$ & $\begin{array}{l}18 \cdot 0 \\
25 \cdot 7 \\
28 \cdot 3\end{array}$ & $\begin{array}{l}905 \\
206 \\
661\end{array}$ & $\begin{array}{l}28 \cdot 2 \\
33 \cdot 0 \\
34 \cdot 3\end{array}$ & $\begin{array}{l}914 \\
215 \\
671\end{array}$ & $\begin{array}{l}1 \cdot 3 \\
1 \cdot 0 \\
3 \cdot 7\end{array}$ & $\begin{array}{r}1249 \\
306 \\
943\end{array}$ & $\begin{array}{l}17 \cdot 7 \\
19 \cdot 8 \\
26 \cdot 9\end{array}$ & $\begin{array}{r}1010 \\
242 \\
672\end{array}$ & $\begin{array}{l}23 \cdot 4 \\
30 \cdot 9 \\
35 \cdot 3\end{array}$ & $\begin{array}{l}985 \\
249 \\
712\end{array}$ \\
\hline $\begin{array}{l}\text { Father against smoking } \\
\text { Father favours smoking }\end{array}$ & $\begin{array}{l}4 \cdot 1 \\
6 \cdot 2\end{array}$ & $\begin{array}{r}763 \\
1514\end{array}$ & $\begin{array}{l}19 \cdot 2 \\
24 \cdot 5\end{array}$ & $\begin{array}{r}577 \\
1117\end{array}$ & $\begin{array}{l}27 \cdot 4 \\
33 \cdot 3\end{array}$ & $\begin{array}{r}610 \\
1127\end{array}$ & $\begin{array}{l}1 \cdot 7 \\
2 \cdot 3\end{array}$ & $\begin{array}{r}818 \\
1564\end{array}$ & $\begin{array}{l}17 \cdot 5 \\
23 \cdot 7\end{array}$ & $\begin{array}{r}664 \\
1179\end{array}$ & $\begin{array}{l}26 \cdot 0 \\
30 \cdot 7\end{array}$ & $\begin{array}{r}649 \\
1227\end{array}$ \\
\hline $\begin{array}{l}\text { Mother against smoking } \\
\text { Mother favours smoking }\end{array}$ & $\begin{array}{l}3.0 \\
7.9\end{array}$ & $\begin{array}{l}1183 \\
1224\end{array}$ & $\begin{array}{l}20 \cdot 6 \\
25 \cdot 4\end{array}$ & $\begin{array}{l}884 \\
913\end{array}$ & $\begin{array}{l}28 \cdot 3 \\
34 \cdot 2\end{array}$ & $\begin{array}{l}905 \\
916\end{array}$ & $\begin{array}{l}1 \cdot 7 \\
2 \cdot 7\end{array}$ & $\begin{array}{l}1241 \\
1276\end{array}$ & $\begin{array}{l}18 \cdot 2 \\
24 \cdot 3\end{array}$ & $\begin{array}{l}982 \\
957\end{array}$ & $\begin{array}{l}25 \cdot 1 \\
32 \cdot 5\end{array}$ & $\begin{array}{l}973 \\
992\end{array}$ \\
\hline $\begin{array}{l}\text { Father against youth smoking } \\
\text { Father favours youth smoking }\end{array}$ & $\begin{array}{l}5 \cdot 3 \\
6 \cdot 5\end{array}$ & $\begin{array}{r}1952 \\
325\end{array}$ & $\begin{array}{l}22 \cdot 9 \\
21 \cdot 5\end{array}$ & $\begin{array}{r}1448 \\
246\end{array}$ & $\begin{array}{l}30 \cdot 9 \\
33 \cdot 2\end{array}$ & $\begin{array}{r}1493 \\
244\end{array}$ & $\begin{array}{l}1 \cdot 9 \\
3 \cdot 0\end{array}$ & $\begin{array}{r}2013 \\
365\end{array}$ & $\begin{array}{l}21 \cdot 2 \\
23 \cdot 3\end{array}$ & $\begin{array}{r}1561 \\
279\end{array}$ & $\begin{array}{l}28 \cdot 4 \\
33 \cdot 3\end{array}$ & $\begin{array}{r}1559 \\
273\end{array}$ \\
\hline $\begin{array}{l}\text { Mother against youth smoking } \\
\text { Mother favours youth smoking }\end{array}$ & $\begin{array}{l}5 \cdot 0 \\
8 \cdot 8\end{array}$ & $\begin{array}{r}2102 \\
308\end{array}$ & $\begin{array}{l}22 \cdot 1 \\
30 \cdot 4\end{array}$ & $\begin{array}{r}1582 \\
214\end{array}$ & $\begin{array}{l}30 \cdot 0 \\
39 \cdot 9\end{array}$ & $\begin{array}{r}1598 \\
223\end{array}$ & $\begin{array}{l}2 \cdot 1 \\
3 \cdot 2\end{array}$ & $\begin{array}{r}2143 \\
374\end{array}$ & $\begin{array}{l}21 \cdot 3 \\
21 \cdot 1\end{array}$ & $\begin{array}{r}1674 \\
265\end{array}$ & $\begin{array}{l}28 \cdot 2 \\
32 \cdot 6\end{array}$ & $\begin{array}{r}1698 \\
267\end{array}$ \\
\hline $\begin{array}{l}\text { Father against smokers' rights } \\
\text { Father favours smokers' rights }\end{array}$ & $\begin{array}{l}4 \cdot 7 \\
5 \cdot 9\end{array}$ & $\begin{array}{r}786 \\
1487\end{array}$ & $\begin{array}{l}21 \cdot 0 \\
23 \cdot 6\end{array}$ & $\begin{array}{r}587 \\
1105\end{array}$ & $\begin{array}{l}32 \cdot 0 \\
30 \cdot 7\end{array}$ & $\begin{array}{r}606 \\
1129\end{array}$ & $\begin{array}{l}1 \cdot 5 \\
2 \cdot 4\end{array}$ & $\begin{array}{r}791 \\
1588\end{array}$ & $\begin{array}{l}18 \cdot 2 \\
23 \cdot 1\end{array}$ & $\begin{array}{r}610 \\
1230\end{array}$ & $\begin{array}{l}25 \cdot 8 \\
30 \cdot 8\end{array}$ & $\begin{array}{r}620 \\
1254\end{array}$ \\
\hline $\begin{array}{l}\text { Mother against smokers' rights } \\
\text { Mother favours smokers' rights }\end{array}$ & $\begin{array}{l}4 \cdot 5 \\
6 \cdot 1\end{array}$ & $\begin{array}{r}829 \\
1577\end{array}$ & $\begin{array}{l}19 \cdot 9 \\
24 \cdot 8\end{array}$ & $\begin{array}{r}628 \\
1167\end{array}$ & $\begin{array}{l}31 \cdot 5 \\
31 \cdot 1\end{array}$ & $\begin{array}{r}644 \\
1174\end{array}$ & $\begin{array}{l}1 \cdot 3 \\
2 \cdot 7\end{array}$ & $\begin{array}{r}874 \\
1637\end{array}$ & $\begin{array}{l}19 \cdot 3 \\
22 \cdot 4\end{array}$ & $\begin{array}{r}696 \\
1239\end{array}$ & $\begin{array}{l}26 \cdot 3 \\
30 \cdot 2\end{array}$ & $\begin{array}{r}688 \\
1274\end{array}$ \\
\hline $\begin{array}{l}\text { Father favours smoking prevention } \\
\text { Father against smoking prevention }\end{array}$ & $\begin{array}{l}5 \cdot 3 \\
7 \cdot 6\end{array}$ & $\begin{array}{r}2140 \\
132\end{array}$ & $\begin{array}{l}22 \cdot 5 \\
25 \cdot 7\end{array}$ & $\begin{array}{r}1588 \\
105\end{array}$ & $\begin{array}{l}31 \cdot 3 \\
30 \cdot 9\end{array}$ & $\begin{array}{r}1641 \\
94\end{array}$ & $\begin{array}{l}2 \cdot 2 \\
6 \cdot 7\end{array}$ & $\begin{array}{r}2220 \\
152\end{array}$ & $\begin{array}{l}21 \cdot 8 \\
17 \cdot 0\end{array}$ & $\begin{array}{r}1730 \\
106\end{array}$ & $\begin{array}{l}29 \cdot 2 \\
28 \cdot 3\end{array}$ & $\begin{array}{r}1754 \\
113\end{array}$ \\
\hline $\begin{array}{l}\text { Mother favours smoking prevention } \\
\text { Mother against smoking prevention }\end{array}$ & $\begin{array}{l}5 \cdot 5 \\
7 \cdot 2\end{array}$ & $\begin{array}{r}2275 \\
125\end{array}$ & $\begin{array}{l}23 \cdot 0 \\
23 \cdot 9\end{array}$ & $\begin{array}{r}1705 \\
88\end{array}$ & $\begin{array}{l}31 \cdot 6 \\
22 \cdot 2\end{array}$ & $\begin{array}{r}1735 \\
81\end{array}$ & $\begin{array}{l}2 \cdot 3 \\
0 \cdot 7\end{array}$ & $\begin{array}{r}2374 \\
134\end{array}$ & $\begin{array}{l}21 \cdot 3 \\
20 \cdot 6\end{array}$ & $\begin{array}{r}1828 \\
102\end{array}$ & $\begin{array}{l}29 \cdot 1 \\
24 \cdot 7\end{array}$ & $\begin{array}{r}1866 \\
93\end{array}$ \\
\hline Smokers & $5 \cdot 9$ & 2407 & $23 \cdot 0$ & 1795 & $31 \cdot 2$ & 1823 & $2 \cdot 2$ & 2520 & $21 \cdot 3$ & 1942 & $28 \cdot 8$ & 1967 \\
\hline
\end{tabular}

to some children describing their parents as smokers because they smoked once or twice a year. More sensitive questioning would determine if this was the case. However, from the extent of agreement between children and parents we are confident that the parents' report, which was used in subsequent analyses, is a reasonable estimate of parental smoking, perhaps missing some parents who smoked very rarely.

Although it was not possible to make directly equivalent comparisons between the parents' reported attitudes and those perceived by the children, there was a large degree of agreement between children and parents in reporting the parents' attitude to the children smoking. Most parents who did not want their children to smoke were perceived as anti-smoking by their children. However, this was not always the case. Roughly a quarter of the parents who claimed that they were against their children smoking were not described as anti-smoking by those children. This may well be a reflection of developmental changes. During adolescence parents gradually relinquish control over their children. ${ }^{10}$ It may be that while many parents would not like to see their children smoking they are reluctant actively to discourage it. Our analysis of change in mothers' attitudes during early $\frac{}{\mathbb{D}}$ adolescence supports this interpretation. During that period there was a movement towards a more permissive view of smoking by the mothers. It seems that as their children develop mothers become more reluctant to attempt to restrict their smoking as part of a general relaxation of controls.

Both children and parents in one-parent families were more likely to smoke. Such families are known to endure a variety of social and economic problems. ${ }^{11}$ The consequent higher level of stress seems to be conducive to smoking. Further, it has been shown that when one member of a family ceases $\frac{\text { 의 }}{2}$ to play his or her rôle for any reason (eg, divorce, $\rightarrow$ sickness, death), then other family members take over the vacant rôle to ensure that the family unit N continues to function. ${ }^{12}$ By taking on additional rôle responsibility the individual can develop a variety of $N$ physical and psychological problems. Our finding $\omega$ that the boys without a father and the girls without a 
Table 6 Proportion of adolescents smoking in 1978 and 1981 in relation to changing characteristics of their parents between 1974 and 1978

\begin{tabular}{|c|c|c|c|c|c|c|c|c|}
\hline \multirow[b]{3}{*}{ Changing parental characteristics $1974-8$} & \multicolumn{4}{|l|}{ Boys } & \multicolumn{4}{|l|}{ Girls } \\
\hline & \multicolumn{2}{|c|}{$1978(15-16 y r)$} & \multicolumn{2}{|c|}{$1981(18-19 y r)$} & \multicolumn{2}{|c|}{$1978(15-16 y r)$} & \multicolumn{2}{|c|}{$1981(18-19 \mathrm{yr})$} \\
\hline & $\%$ & No & $\%$ & No & $\%$ & No & $\%$ & No \\
\hline Father does not smoke & $14 \cdot 9$ & 664 & $21 \cdot 2$ & 585 & $14 \cdot 5$ & 763 & $21 \cdot 2$ & 664 \\
\hline Father stops smoking & $20 \cdot 0$ & 150 & $23 \cdot 4$ & 124 & $21 \cdot 2$ & 170 & 19.9 & 151 \\
\hline Father adopts smoking & $15 \cdot 0$ & 40 & $30 \cdot 3$ & 33 & $14 \cdot 9$ & 47 & $24 \cdot 4$ & 41 \\
\hline Father smokes throughout & $24 \cdot 6$ & 496 & $34 \cdot 1$ & 419 & $22 \cdot 3$ & 539 & $27 \cdot 8$ & 453 \\
\hline Mother does not smoke & $16 \cdot 3$ & 858 & $24 \cdot 5$ & 734 & $15 \cdot 7$ & 996 & $19 \cdot 9$ & 861 \\
\hline Mother stops smoking & $20 \cdot 5$ & 88 & $21 \cdot 8$ & 78 & $20 \cdot 0$ & 70 & $30 \cdot 5$ & 59 \\
\hline Mother adopts smoking & $30 \cdot 6$ & 49 & $35 \cdot 6$ & 45 & $23 \cdot 5$ & 51 & $38 \cdot 5$ & 39 \\
\hline Mother smokes throughout & $24 \cdot 5$ & 364 & $30 \cdot 3$ & 307 & $22 \cdot 5$ & 417 & $28 \cdot 9$ & 360 \\
\hline Mother against smoking & $15 \cdot 6$ & 488 & $21 \cdot 2$ & 424 & $15 \cdot 2$ & 580 & $18 \cdot 6$ & 500 \\
\hline Mother moves against smoking & $19 \cdot 8$ & 202 & $23 \cdot 7$ & 173 & $20 \cdot 4$ & 245 & 25.9 & 212 \\
\hline Mother moves in favour of smoking & $19 \cdot 1$ & 220 & 28.9 & 190 & $17 \cdot 3$ & 243 & $22 \cdot 7$ & 207 \\
\hline Mother favours smoking & $23 \cdot 1$ & 472 & $31 \cdot 3$ & 396 & $20 \cdot 9$ & 487 & $28 \cdot 1$ & 416 \\
\hline Mother against youth smoking & $18 \cdot 7$ & 1215 & $25 \cdot 9$ & 1035 & $17 \cdot 9$ & 1358 & $23 \cdot 5$ & 1181 \\
\hline Mother moves against youth smoking & $20 \cdot 0$ & 50 & $27 \cdot 5$ & 51 & $19 \cdot 7$ & 61 & $23 \cdot 4$ & 47 \\
\hline Mother moves in favour of youth smoking & $22 \cdot 4$ & 98 & $28 \cdot 8$ & 80 & $17 \cdot 0$ & 100 & $22 \cdot 9$ & 83 \\
\hline Mother favours youth smoking & $33 \cdot 3$ & 9 & $20 \cdot 0$ & 10 & $29 \cdot 6$ & 27 & $22 \cdot 2$ & 18 \\
\hline Mother against smokers' rights & $15 \cdot 5$ & 219 & $24 \cdot 7$ & 194 & $10 \cdot 8$ & 260 & $20 \cdot 3$ & 222 \\
\hline Mother moves against smokers' rights & $15 \cdot 5$ & 187 & $20 \cdot 8$ & 149 & $16 \cdot 3$ & 202 & $23 \cdot 3$ & 172 \\
\hline Mother moves in favour of smokers' rights & $17 \cdot 6$ & 267 & $29 \cdot 2$ & 233 & $19 \cdot 3$ & 316 & $22 \cdot 4$ & 272 \\
\hline Mother favours smokers' rights & $22 \cdot 2$ & 703 & $27 \cdot 2$ & 603 & 20.7 & 774 & 25.0 & 668 \\
\hline Mother favours smoking prevention & $19 \cdot 4$ & 792 & $26 \cdot 3$ & 692 & $18 \cdot 4$ & 860 & $23 \cdot 5$ & 745 \\
\hline Mother moves to favour smoking prevention & $19 \cdot 0$ & 147 & $26 \cdot 8$ & 123 & $19 \cdot 3$ & 161 & $15 \cdot 9$ & 126 \\
\hline Mother moves against smoking prevention & $19 \cdot 0$ & 252 & $29 \cdot 2$ & 209 & 16.7 & 299 & $27 \cdot 4$ & 266 \\
\hline Mother against smoking prevention & $21 \cdot 2$ & 170 & $21 \cdot 4$ & 140 & $18 \cdot 6$ & 220 & $22 \cdot 2$ & 185 \\
\hline
\end{tabular}

mother were most likely to smoke suggests that these teenagers adopt smoking to cope with the strain of filling the rôle of the missing same-sex parent.

Our results confirm that, in general, parental smoking behaviour is more important than parental attitudes in the development of smoking among children. The clear sex-linking in the relation between parents' and children's smoking behaviour suggests that to many adolescents the uptake of smoking is part of the process of becoming adult as modelled by the same-sex parent. Even among the older adolescents this influence of parental smoking was still apparent. Further, when the parents changed their smoking behaviour the risk of their children smoking altered accordingly. This agrees with the findings in Horn's original study. ${ }^{1}$

Although the fathers' attitudes to smoking were not significantly related to the smoking behaviour of their children, there was a significant relation between mothers' attitudes and the boys' smoking. The smaller influence of fathers' attitudes probably reflects their lesser rôle in child-rearing. The importance of the mothers' attitudes for the boys but not for the girls is interesting. According to the children's own reports the mothers were less likely to discourage smoking among boys than among girls (table 3). Not only did the mothers have a different attitude to boys smoking, but the boys themselves seemed to be more influenced by their mothers' attitudes.

In conclusion, our findings confirm that parental smoking behaviour and attitudes are an influential factor in the development of smoking among adolescents. One hopeful sign is that by giving up smoking or changing their view of children smoking the parents seemed to reduce the risk of their children smoking. This is a factor that should be considered in the design of future health education programmes for children. Research conducted in Norway ${ }^{13}$ found that the effectiveness of school anti-smoking programmes was greatly increased when the parents were involved.

Previous anti-smoking campaigns have concentrated much of their effort on discouraging children from smoking with the aim of creating a non-smoking generation. This approach does not take sufficient account of the fact, reconfirmed by our findings, that children are influenced by the behaviour and attitudes of adults, especially their parents. The most effective way to stop children smoking may be to discourage smoking among adults. For example, the decline in the prevalence of 
smoking among middle class adults over the past 30 years has been followed by the emergence of a social class gradient in the prevalence of smoking among children. ${ }^{14}$ Future health education efforts should not consider children in isolation but rather as part of a society in which smoking is still acceptable adult behaviour. Unless action is taken on a societal level to reduce smoking among adults then the aim of creating a non-smoking generation will be frustrated.

\section{References}

${ }^{1}$ Horn D, Courts FA, Taylor RM, Solomon ES. Cigarette smoking among high-school students. Amer J Publ Health 1959; 49: 1497-1511.

${ }^{2}$ Nilsen E. Smoking habits among school children in Norway. Br J Prev Soc Med 1959; 12: 135-13.

${ }^{3}$ Bewley BR, Bland JM. Academic performance and social factors related to cigarette smoking by school children. Br J Prev Soc Med 1977; 31: 18-24.

${ }^{4}$ Palmer, AB. Some variables contributing to the onset of cigarette smoking among junior, high school students. Soc Sci Med 1970; 4:359-66.
${ }^{5}$ Nolte AE, Smith BJ, O'Rouke T. The relative importance of parental attitudes and behaviour upon youth smoking behaviour. J School Health 1983; 53: 264-71.

${ }^{6}$ Baric L, McArthur C, Fisher C. Norms, attitudes and smoking behaviour among Manchester students. Health Educ J 1976; 35: 142-52.

${ }^{7}$ Murray M, Swan AV, Bewley BR, Johnson MRD. The development of smoking during adolescence-The MRC/Derbyshire Smoking Study. Inter J Epidemiol 1983; 12: 185-92.

${ }^{8}$ Baker RT, Nelder JA. The GLIM Manual for System 3. Oxford: Numerical Algorithms Group, 1978.

${ }^{9}$ Murray M, Swan AV, Johnson MRD, Bewley BR. Some factors associated with increased risk of smoking by children. J Child Psychol Psychiat 1983; 24: 223-32.

${ }^{10}$ Bandura A. The stormy decade: fact or fiction? In: Rogers D, ed. Issues in adolescent psychology (2nd ed). New York: Appleton-Century-Croft, 1972 .

${ }^{11}$ Ferri E. Growing up in a one-parent family. Windsor: NFER, 1976.

${ }^{12}$ Wilson C. The family. In: Camberwell Council on Alcoholism, Women and alcohol. London: Tavistock, 1980.

${ }^{13}$ Aaro LE, Bruland E, Hauknes A, Locksen PM. Smoking among Norwegian schoolchildren 1975-1980. III The effects of anti-smoking campaigns. Scand J Psychol 1983; 24: 277-83.

${ }^{14}$ Johnson MRD, Murray M, Bewley BR, Clyde DC, Banks MH, Swan AV. Social class, parents, children and smoking. Bull Inter Union Ag Tubercul 1982; 57. 258-62. 